\title{
On Cotnoir's two notions of proper parthood
}

\author{
Massimiliano Carrara $^{1} \cdot$ Jeroen Smid $^{2}$ (1)
}

Accepted: 11 January 2022/Published online: 30 January 2022

(C) The Author(s) 2022

\begin{abstract}
A.J. Cotnoir has argued that we should distinguish between two notions of proper parthood: outstripped part and non-identical part. Outstripped parthood is an asymmetric relation, but non-identical parthood is not. We argue, first, that the intuitions Cotnoir uses to motivate these notions do not always give the right verdict; and, second, that systematic reasons for distinguishing these two notions of parthood have further counter-intuitive consequences. This means the distinction between two notions of proper parthood currently lacks adequate motivation.
\end{abstract}

Keywords Parthood - Proper part - Non-identical part · Outstripped part · Mereology

\section{Introduction}

In a number of publications A.J. Cotnoir (2010, 2016a, b, 2018) has argued for distinguishing two notions of proper parthood: outstripped part and non-identical part. An example of an outstripped part is the head of the statue David in relation to

Jeroen would like to thank the Swedish Research Council (VR, international postdoc grant number 2017-06160-3) and the Netherlands Organisation for Scientific Research (NWO, project number VI.Veni.201F.006: The Whole Explanation, Part by Part) for funding his research.

Jeroen Smid

j.smid@uva.nl

Massimiliano Carrara

massimiliano.carrara@unipd.it

1 Department of Philosophy, Sociology, Education and Applied Psychology (FISPPA),

University of Padua, Padua, Italy

2 Department of Philosophy; Institute for Logic, Language and Computation (ILLC), University of Amsterdam, Amsterdam, The Netherlands 
David. While an example of a non-identical part is the marble that coincides with David in relation to David—as well as David in relation to its marble. So something is an outstripped part of something else, if and only if the former is part of the latter, but the latter is not part of the former. And something is a non-identical part of something else if and only if it is a part of it but not identical with it. A non-identical part can coincide with the whole of which it is a non-identical part, while an outstripped part always leaves a remainder if taken away from the whole. The difference between these two notions of proper part thus only matters for a nonextensional mereology. Although David and its marble have all the same outstripped parts, they are not identical. Hence, according to Cotnoir, David and its marble are non-identical parts of each other. An extensional mereologist, however, does not recognise the difference between outstripped part and nonidentical part: no two distinct objects can have exactly the same outstripped parts.

Two intuitions can be traced in Cotnoir's work that motivate the distinction between outstripped part and non-identical part. The first is that whenever an outstripped part is taken away from a whole it leaves a remainder, but taking away a non-identical part does not always leave a remainder. The second is that an outstripped part is mereologically distinguishable from its whole but a non-identical part is not. An additional reason Cotnoir gives for distinguishing non-identical part from outstripped part concerns the harmony between the mereological structure of objects and the mereological structure of their locations.

(Another reason to distinguish the two notions of proper parthood is, obviously, that it allows for a non-extensional mereology. However, it is not the only way to allow for a non-extensional mereology. For example, a mereology with a single asymmetric notion of proper parthood but without the principle Strong Supplementation (see below) is non-extensional. See, for example, Simons (1987, 26ff).) We consider it more fruitful to engage directly with the (other) motivations Cotnoir presents behind his distinction than to argue for extensional mereology because even a non-extensional mereologist might not want to distinguish between outstripped and non-identical part.)

In Sect. 2 we provide definitions of all the relevant parthood relations in terms of a primitive notion (overlap) that does not beg the question to either friends or foes of the distinction between outstripped and non-identical part. We also show which principles hold for which notions and explain some interrelations. We then argue in Sect. 3 that the intuitions behind the distinction fail to properly motivate the distinction. The first intuition backfires because there are scenarios where Cotnoir's account tells us that we have non-identical parts, but taking a part away would leave a remainder-signalling that this is instead a case of outstripped part. The second intuition, we argue, is a non-starter because examples of objects that are qualitatively different while mereological indiscernible are self-refuting at best, and question-begging at worst.

Cotnoir's systematic reason for employing non-identical parts alongside outstripped parts concerns co-location. We discuss this in Sect. 4. Unfortunately, this reason is directly connected, again, to intuitions about remainders. We argue against this systematic reason by giving another example of co-location that, for us, triggers the remainder intuition. 
We conclude that we have no good reasons to distinguish outstripped from nonidentical part since the intuitions guiding this distinction result in counter-intuitive solutions to some co-location scenarios.

\section{A neutral basis}

In order to properly assess whether we should distinguish non-identical part from outstripped part, it is best to give both notions a definition in terms of a primitive that both friends and foes of the distinction agree upon and within a mereological theory that is consistent with the distinction. We can then distinguish these two notions with respect to the formal principles they obey. So, following Cotnoir (2016a), we take 'overlap' (and 'is identical with') as primitive and define the following notions.
D1 $x \leq y={ }_{d f} \forall z(z \circ x \rightarrow z \circ y)$
D2 $x \leq y={ }_{d f} x \leq y \wedge x \neq y$
D3 $x<y={ }_{d f} x \leq y \wedge \neg y \leq x$
D4 $F u\left(y, \varphi_{x}\right)=_{d f} \forall z\left(z \circ y \leftrightarrow\left(\exists x\left(\varphi_{x} \wedge z \circ x\right)\right)\right)$

(Parthood)

(Non-Identical Parthood)

(Outstripped Parthood)

(Fusion)

Note that the first definition ensures, by pure logic, that parthood is reflexive and transitive. Also, it should be clear that from the logic of identity and the definitions it follows that every outstripped part is also a non-identical part. The converse, however, only holds if we have the anti-symmetry of parthood, i.e. the principle stating that if $x$ and $y$ are parts of each other, then $x=y$.

Any mereology without anti-symmetry is by definition a non-well-founded mereology, which can be axiomatized by two axioms:

P1 $\quad x \circ y \leftrightarrow \exists z(z \leq x \wedge z \leq y)$

(Shared Part)

P2 $\exists x \varphi_{x} \rightarrow \exists y F u\left(y, \varphi_{x}\right)$

(Unrestricted Composition)

(Note that these principles establish, inter alia, that overlap is reflexive and symmetric.)

To turn the theory based on these two axioms into classical mereology — which is well-founded-we would only need to add the following:

P3 $\forall z(z \circ x \leftrightarrow z \circ y) \rightarrow x=y$

(Overlap Extensionality)

But once Overlap Extensionality is added, there is no longer any difference between Non-Identical Parthood and Outstripped Parthood. For if $x$ is a nonidentical part of $y$ then by definition $x \neq y$. Hence, by the contrapositive of Overlap Extensionality, one of $x$ and $y$ should overlap something that the other does not overlap. By the definition of parthood this means that either $x$ is part of $y$ but $y$ is not part of $x$, or vice versa. But that is just to say that one of them is an outstripped part of the other. Hence we refrain from adding Overlap Extensionality to the axiomatic base.

However, a principle very similar to it, commonly called 'Strong Supplementation', is a direct consequence of D1: 
That brings us to one of the key issues, the cousins of Strong Supplementation:

P5 $x \leq y \rightarrow \exists z(z \leq y \wedge \neg z \circ x)$

P6 $x<y \rightarrow \exists z(z \leq y \wedge \neg z \circ x)$
(Non-Identical Supplementation) (Outstripped Supplementation)

P5 and P6 are often conflated and called 'Weak Supplementation'. But if we distinguish between the two notions of proper parthood, we should distinguish between P5 and P6. (As already noted by Maureen Donnelly (2011, 234ff).) Cotnoir 2018 convincingly argues that $\mathbf{P 6}$ is analytic, i.e. true in virtue of its meaning. One of his arguments is that the definition of being an outstripped part entails the consequent of outstripped supplementation.

Again, we should refrain from adding P5 if we want to distinguish non-identical part from outstripped part. To see this, consider a case where $x$ is a non-identical part of $y$ but not an outstripped part. This means that $y$ is also a non-identical part of $x$. By two application of P5 we then get that $x$ should have a part disjoint from $y$ and $y$ should have a part disjoint from $x$. This means that neither is part of the other (by D1)_contradiction.

Let us highlight some of the formal features of 'pure' non-identical part, by which we mean the case where $x \leq y$ and $\neg x<y$. (These are 'pure' in the sense that the non-identity is not a consequence of the fact that $x$ is an outstripped part of $y$.) Pure non-identical parthood is non-reflexive and symmetric, thus not transitive. (Unless, of course, it were vacuously symmetric in which case it could be vacuously transitive.) The failure of transitivity simply follows from the fact that the relation is non-reflexive and symmetric. To be sure, pure non-identical part is transitive in the cases where $x, y$, and $z$ are all distinct. (From hereon we use 'non-identical part' to mean 'pure non-identical part', unless stated otherwise.)

So, non-identical parthood is symmetric, does not satisfy P5 (i.e., its version of Weak Supplementation), and is not transitive. This means that it fails every one of the conditions - i.e., transitivity, asymmetry, and weak supplementation - that Peter Simons (1987, p. 362) considers "the minimum we can require of a relation if it is to be one of proper part to whole." (Note that Simons favours a non-extensional mereology.) Similarly, Walters (2017) argues that symmetric parthood is not really a proper parthood relation.

We agree with this insofar that many people think of outstripped part rather than non-identical part when they use 'part of' and cognate terms. For example, 'being simple' is commonly understood as 'lacking proper parts', and 'lacking proper parts' seems to be understood as 'lacking outstripped parts' and not merely as 'lacking non-identical parts'. However, this does not mean there is no distinction to be drawn between non-identical part and outstripped part. At most it gives a reason to think that the name 'non-identical part' is inapt because we commonly associate with 'part' certain formal features that this relation does not exhibit. But we could name the relation differently (for example, as 'is coincident with'). For uniformity we stick with 'non-identical part'.

So it is clear that outstripped part and non-identical part can be distinguished with respect to which formal principles they obey. The key question is whether this 
formal difference corresponds to a difference in the world. One way to assess this is to consider the intuitions behind the distinction: are these any good?

\section{The intuitions behind weak supplementation apply to both parthood relations}

One key difference between outstripped part and non-identical part is that only the former satisfies Weak Supplementation. Cotnoir is quite explicit about which intuitions lie behind Weak Supplementation and, hence, should guide our thinking about the notion of outstripped part.

He writes that the "idea is, roughly, that whenever an object is a proper [outstripped] part of another, then subtracting that part must leave something (or things) of the original object behind - there must be some remainder." (Cotnoir $2018,7)$ So the first intuition behind outstripped part is that you can "take it away" from a whole without taking the whole away completely.

A second intuition is that in the case of an outstripped part "there's a mereological and not merely qualitative difference between an object and its proper part." (Cotnoir 2018, 9) Thus the idea here is that in the case of an outstripped part we can point to some mereological feature that is only had by the outstripped part or only had by the whole.

We take it that non-identical part is meant to satisfy the opposite of these two intuitions. Thus, a non-identical part of a whole cannot be taken away while leaving a remainder and it has all and only the mereological features had by the whole.

Now, Cotnoir often suggests that objects are non-identical parts of each other if they are co-located. (Cotnoir 2010, 401; Cotnoir 2016a, 963; Cotnoir 2016b, 130 fn.17) (More about co-location in the next section.) But some co-located objects can satisfy the remainder intuition and should thus be taken as outstripped parts rather than non-identical parts. Here is one scenario that suggests that we can have a remainder while taking away a non-identical part. Consider the classic case of a statue and its matter, and suppose that they are indeed co-located, i.e. numerically distinct yet occupying the exact same region. It seems that we can "take away" the statue from the matter while leaving the matter intact, for example by destroying the statue in such a way that leaves the constituting matter intact. This thus suggests that the statue is an outstripped part of its matter instead of a non-identical partcontrary to what Cotnoir suggests.

The statue and the clay are, arguably, of different ontological kinds. So a defender of the distinction between outstripped and non-identical part could respond that an outstripped part of an object is always of the same kind as that of which it is part. (We note that Cotnoir does not suggest this.) Now, some friends of co-location think that even entities of the same kind are co-located (Fine 2000; Hershenov 2003; Spolaore 2012). Consider, for example, a case from Giuseppe Spolaore (2012) where a rope is made out of threads of cotton and a net is made out of the rope. Spolaore holds that the net and the rope are co-located entities belonging to the same ontological kind. Moreover, it seems we can take away the net without taking away the rope by taking the net apart. So the net seems to be an outstripped part of 
the rope rather than a non-identical part-contrary to the claim that co-located entities are non-identical parts of each other.

However, maybe these two examples misconstrue the intended notion of "taking away". Taking away the top of a pencil by breaking it off is arguably a different form of "taking away" a statue from a piece of clay by reshaping the latter (and different from taking apart a net while leaving the rope intact). But even if we limit ourselves to the "breaking off" form of taking away, we can think of scenario's where co-located objects satisfy the remainder intuition.

For example, consider three numerically distinct objects, $b_{1}, b_{2}$, and $b_{3}$; and their fusion, $x$. Say that at $t_{1}$ the $b_{i}$ 's do not overlap and are not co-located. In that case each of the $b_{i}$ 's is an outstripped part of $x$ and each can thus be taken away from $x$ while leaving a remainder. Now, say that at $t_{2}, b_{1}, b_{2}$, and $b_{3}$ are all co-located. Then, according to Cotnoir, at $t_{2}$ each of the $b_{i}$ 's is a non-identical part of $x$ and $x$ is a non-identical part of each of the $b_{i}$ 's. However, it seems to us that each one of the $b_{i}$ 's can still be taken away from $x$ while leaving a remainder. Our intuition is based on the fact that before $t_{2}$ the objects were outstripped parts of $x$ : one of them could be taken away while leaving a remainder. The mere fact that the parts of $x$ became co-located does not seem to alter this fact. Thus, each of them should still be an outstripped part of $x$. But none of the $b_{i}$ 's can, according to Cotnoir, be an outstripped part of $x$ because $x$ is a non-identical part of each of the $b_{i}$ 's (since they are all co-located).

One could respond that at $t_{2}$ these four objects cannot be co-located because colocation is impossible, or at least co-location as a result of interpenetration. However, Cotnoir seems to allow for co-location due to interpenetration so our description of what goes on at $t_{2}$ is not question-begging. Indeed, if Simons (1994, 571) and Hawthorne and Uzquiano (2016, 55-56) are right that distinct bosons may be co-located, then the last scenario above is physically possible.

Note, moreover, that one cannot respond by saying that each of $b_{1}, b_{2}, b_{3}, x$ are both (impure!) non-identical parts and outstripped parts of each other. For if $x$ is an outstripped part of $b_{1}$ (say), then taking away $x$ from $b_{1}$ should leave a remainder. But since $x$ is a fusion of $b_{1}, b_{2}$, and $b_{3}$; taking away $x$ from $b_{1}$ cannot leave a remainder. The same holds for the $b_{i}$ 's. One might think that $b_{1}$ is both an (impure!) non-identical part and outstripped part of $b_{2}$ (say). But then $b_{2}$ should also be an outstripped and (impure!) non-identical part of $b_{1}$, because $b_{1}$ and $b_{2}$ are co-located and there is no reason to hold that only one of them is an outstripped part of the other. But now we end up with a contradiction, since outstripped part is asymmetric.

So the first intuition does little to distinguish outstripped from non-identical part. But we still have the second intuition, i.e. that outstripped parts are mereologically distinguishable, whereas non-identical parts are mereologically indistinguishable. However, it is hard to turn this into a robust test, for two reasons. First, the usual arguments for the claim that there are qualitative differences without a mereological difference are the classical arguments against extensional mereology. A problem with these arguments is that they presuppose what is to be demonstrated, namely, that there are different objects although they have the same mereological features (Lewis, 1971, 204ff; Jubien, 1993, 118ff; Varzi, 2000, 291ff). 
Second, as Achille Varzi has argued, examples where $x$ and $y$ are taken to be qualitatively different but mereologically indistinguishable are self-defeating. Because the reasons for distinguishing $x$ and $y$ may also be employed to distinguish an outstripped part of $x$ from a co-located outstripped part of $y$. For example, a statue may be distinct from its marble because the statue is made by an artist and the marble not. But, similarly, the head of the statue is made by an artist, while its portion of marble is not. This means that a good case can be made that the statue and the marble are mereologically distinguishable after all: the head is part of statue but, it seems, not part of the marble (Varzi 2008).

Finally, and as said in the introduction, non-extensional mereologies can be had without distinguishing between non-identical and outstripped part. As such, we do not think it is fruitful to discuss this second intuition any further here: if one thinks a qualitative difference without a mereological difference is impossible, then one is welcome to join the extensional mereologists. If one instead thinks some objects are mereologically indiscernible but qualitatively discernible, then one is at home with the non-extensionalists. But being a non-extensionalist does not, by itself, warrant a distinction between outstripped and non-identical part. (Of course, another option at this point would be to debate the evidential status of intuitions, but that goes well beyond the scope of this paper.)

\section{Co-location and harmony}

Fortunately, Cotnoir does not base his account on intuitions alone. Instead, he gives a systematic reason why we should accept the notion of a non-identical part. It concerns the interplay between the mereological structure of an object and the mereological structure of its location. To get clear on this, let us take ' $x @ r$ ' as a primitive notion meaning ' $x$ is exactly located at region $r$ '. This should be understood to hold between an object and the region that it exactly occupies, such that the object has the same volume and stands in the same spatio-temporal relations to other things as the region. (From hereon, variables $x, y, z$ range over objects, and variables $r, s$, and $t$ range over regions of space.)

Cotnoir (2016a, 962ff) is attracted to a mirroring principle according to which parts of objects mirror parts of regions in the following sense:

PL $\forall x \forall y(x \leq y \leftrightarrow \exists r \exists s(x @ r \wedge y @ s \wedge r \leq s))$

(Part Mirroring)

From this principle and the definition of outstripped part, it follows that

OPL $\forall x \forall y(x<y \leftrightarrow \exists r \exists s(x @ r \wedge y @ s \wedge r<s))$

(Outstripped Part Mirroring)

One argument for non-identical parts is that it allows, with the mereology presented in Sect. 2, for both co-location and the harmony principle PL: "it may well be the only co-locational mereology compatible with [this assumption] about location" (Cotnoir 2016a, 963). (Strictly speaking, Cotnoir here refers to a materially equivalent principle but mentions PL in a footnote.)

So it seems the debate should centre around PL; its status is crucial for a proper assessment of the difference between outstripped part and non-identical part. For if 
PL holds, then any reason in favour of co-location is a reason for accepting a notion of non-identical part. Conversely, if $\mathbf{P L}$ is false, then reasons for accepting colocation are not reasons for accepting non-identical parthood.

Unfortunately, it is hard to determine the plausibility of PL independently of the plausibility of the arguments to introduce the notion of non-identical part. The reason is that intuitive cases of non-identical parthood are linked to exact colocation. Intuitions seem to diverge here, independent of considerations concerning extensionality. Consider a scenario where two simple objects (simple in the sense of lacking outstripped parts) are co-located. Cotnoir would say that these simples are then non-identical parts of each other, in line with PL. We think, however, that in the situation described we merely have a case where two objects stand in no mereological relations at all (except for disjointness) even though they have the exact same location. Part of the reason we think this is because it seems that someone could take away one of the simples while leaving the other unaltered. So again the remainder-intuition seems to apply here, meaning that they cannot be nonidentical parts of each other.

With respect to the other intuition, i.e., that there can be a qualitative difference without a mereological difference, Cotnoir's and our verdicts are symmetric in this scenario. We all think these two simples are mereologically indistinguishable. However, we think this is because they have no mereological structure at all, whereas Cotnoir thinks they do have some mereological structure: they are nonidentical parts of each other.

\section{Concluding remarks}

We raised doubts about the notion of non-identical part because intuitions about remainders and about qualitative differences without mereological differences diverge in crucial cases. A harmony principle linking the mereological structure of objects with that of their locations did not provide a conclusive case for nonidentical parts either. The reason was that we could again construct a scenario where we have co-location while also triggering the remainder intuition. So these are not good reasons for distinguishing outstripped from non-identical parts.

We end by noting that there is an indirect argument for the distinction. A mereology satisfying Antisymmetry, Weak Supplementation, and Unrestricted Composition has Overlap Extensionality as a theorem-as well as other extensionality principles (Varzi 2009). But, as Cotnoir (2016b) shows, denying Antisymmetry suffices to block this derivation. But this means one's formal theory recognises two non-equivalent notions of proper parthood. So if one finds both the arguments for Unrestricted Composition and the arguments against extensionality convincing, one ends up with a mereology in which there are two distinct notions of proper parthood.

But the distinction between outstripped and non-identical part is then merely a by-product of the formal theory and does not correspond to any robust intuitions about the way in which objects may be mereologically related to each other. Some mutual parthood theorists may comfortably base their position on this (and hence indirectly on the arguments for Unrestricted Composition and the arguments against 
extensionality). But we suspect many would want the distinction to be warranted even when the arguments for Unrestricted Composition are stripped away.

Acknowledgements The authors would like to thank Aaron Cotnoir, Giorgio Lando, David Liggins, and various anonymous reviewers for valuable comments on previous versions of the paper.

\section{Declarations}

Conflict of interest The authors declare that they have no conflict of interest.

Open Access This article is licensed under a Creative Commons Attribution 4.0 International License, which permits use, sharing, adaptation, distribution and reproduction in any medium or format, as long as you give appropriate credit to the original author(s) and the source, provide a link to the Creative Commons licence, and indicate if changes were made. The images or other third party material in this article are included in the article's Creative Commons licence, unless indicated otherwise in a credit line to the material. If material is not included in the article's Creative Commons licence and your intended use is not permitted by statutory regulation or exceeds the permitted use, you will need to obtain permission directly from the copyright holder. To view a copy of this licence, visit http:// creativecommons.org/licenses/by/4.0/.

\section{References}

Cotnoir, A. J. (2010). Antisymmetry and non-extensional mereology. Philosophical Quarterly, 60(239), 396-405.

Cotnoir, A. J. (2016). How many angels can be in the same place at the same time? A defense of mereological universalism. Mind, 125(500), 959-965.

Cotnoir, A. J. (2016). Does universalism entail extensionalism? Noûs, 50(1), 121-132.

Cotnoir, A. J., 2018, 'Is Weak Supplementation Analytic?', Synthese First Online.

Donnelly, Maureen. (2011). Using mereological principles to support metaphysics. Philosophical Quarterly, 61(243), 225-246.

Fine, Kit. (2000). A counter-example to Locke's thesis. The Monist, 83, 357-361.

Hawthorne, John, \& Uzquiano, Gabriel. (2016). How many angels can dance on the point of a needle? Mind, 120(478), 53-81.

Hershenov, David. (2003). Can there be spatially coincident entities of the same kind? Canadian Journal of Philosophy, 33, 1-22.

Jubien, Michael. (1993). Ontology, Modality, and the Fallacy of Reference. Cambridge: Cambridge University Press.

Lewis, David K. (1971). Counterparts of persons and their bodies. Journal Philosophical, 68, $203-211$.

Simons, Peter M. (1987). Parts: A Study in Ontology. Oxford: Oxford University Press.

Simons, Peter M. (1994). Particulars in particular clothing: three trope theories of substance. Philosophy and Phenomenological Research, 54, 553-575.

Spolaore, Giuseppe. (2012). 'Not Just a Coincidence. Conditional Counter-examples to Locke's Thesis', Thought: A Journal of Philosophy, 1, 108-115.

Varzi, Achille C. (2000). Mereological commitments. Dialectica, 54(4), 283-305.

Varzi, Achille C. (2008). The extensionality of parthood and composition. Philosophical Quarterly, 58(230), 108-133.

Varzi, Achille C. (2009). Universalism entails extensionality. Analysis, 69, 599-604.

Walters, Lee. (2017). Are the statue and the clay mutual parts? Nôis, 53(1), 23-50.

Publisher's Note Springer Nature remains neutral with regard to jurisdictional claims in published maps and institutional affiliations. 